\title{
Differential Selection by Nematodes of an Introduced Biocontrol Fungus vs. Indigenous Fungi in Nonsterile Soil
}

\author{
Tae Gwan Kim ${ }^{1 *}$ and Guy R. Knudsen ${ }^{2}$ \\ ${ }^{1}$ Department of Microbiology, Pusan National University, Pusan 46241, Republic of Korea \\ ${ }^{2}$ Soil and Land Resources Division, Department of Plant, Soil, and Entomological Sciences, University of Idaho, Moscow, ID 83844-2339, USA
}

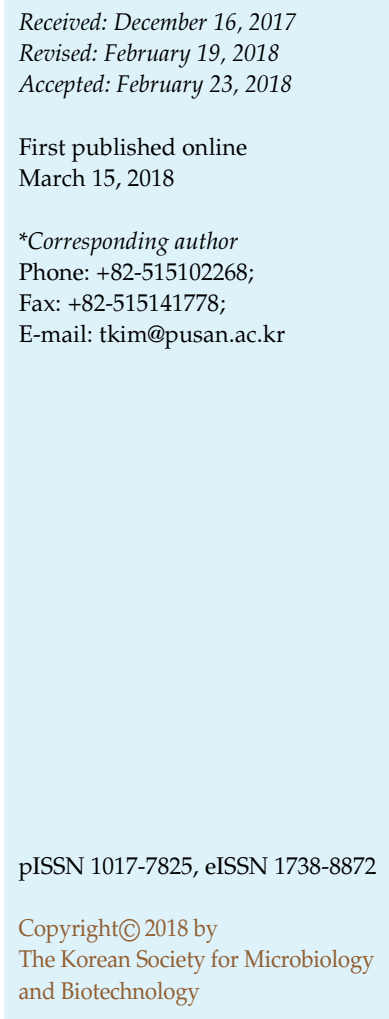

Trophic interactions of introduced biocontrol fungi with soil animals can be a key determinant in the fungal proliferation and activity. This study investigated the trophic interaction of an introduced biocontrol fungus with soil nematodes. The biocontrol fungus Trichoderma harzianum ThzID1-M3 and the fungivorous nematode Aphelenchoides sp. (10 per gram of soil) were added to nonsterile soil, and microbial populations were monitored for 40 days. Similar results were obtained when the experiment was duplicated. ThzID1-M3 stimulated the population growth of indigenous nematodes $(p<0.05)$, regardless of whether Aphelenchoides sp. was added. Without ThzID1-M3, indigenous nematodes did not increase in number and the added Aphelenchoides sp. nematodes almost disappeared by day 10. With ThzID1-M3, population growth of nematodes was rapid between 5 and 10 days after treatment. ThzID1-M3 biomass peaked on day 5, dropped at day 10, and then almost disappeared at day 20, which was not influenced by the addition of nematodes. In contrast, a large quantity of ThzID1-M3 hyphae were present in a heat-treated soil in which nematodes were eliminated. Total fungal biomass in all treatments peaked on day 5 and subsequently decreased. Addition of nematodes increased the total fungal biomass $(p<0.05)$, but ThzID1-M3 addition did not affect the fungal biomass. Hyphae of total fungi when homogenously distributed did not support the nematode population growth; however, hyphae of the introduced fungus did when densely localized. The results suggest that soil fungivorous nematodes are an important constraint on the hyphal proliferation of fungal agents introduced into natural soils.

Keywords: Biocontrol fungus, fungivorous nematode, trophic interaction, differential selection

\section{Introduction}

The use of fungal biocontrol agents can be an important alternative to the use of chemicals for the control of plant pathogens in agriculture. For instance, Trichoderma harzianum has shown biocontrol activity against different phytopathogenic fungi, including Phythium spp., Sclerotium rolfsii, Rhizoctonia solani, Fusarium oxysporum, Sclerotinia sclerotiorum, Botrytis cinerea, and Cladosporium fulvum, in laboratory or field experiments [1-8]. Mycoparasitism has been considered the major mechanism of the antagonistic activity of this fungus. Thus, when such a fungal agent is introduced to soil, the agent must grow and proliferate for effective control of plant pathogens and successful establishment in the environment; for instance, Trichoderma hyphae must grow outward to contact the target propagules of pathogenic fungi in order to initiate mycoparasitism of the propagules $[1,9]$.

Nematodes are probably the most common soil-inhabiting animals, found in nearly all terrestrial ecosystems [10-12]. Free-living nematodes, as bacterivores, fungivores, omnivores, and predators, have key roles in regulating microbial composition, biomass, and activity through their feeding activities and by releasing mineral nutrients [1316]. Fungivorous nematodes feed on mycelia of many different species of soil fungi, including beneficial and pathogenic ones. Fungivores penetrate fungal cell walls with their stylet and remove cytoplasm from the fungal 
cells. For instance, the fungal cytoplasm consumption rate of Aphelenchus avenae is $0.12 \mu \mathrm{g}$ cytoplasm per day [13]. Fungivores usually account for a small portion of the total number of nematodes, and the most common fungivore genera in bulk and agricultural field soils are Aphelenchoides and Aphelenchus [17-19]. Populations of fungivorous nematodes can rapidly increase in natural habitats when suitable fungi are available [14] and environmental conditions are favorable [20]. However, microcosm experiments have shown that, although the presence of filamentous fungi with nutrient sources apparently stimulates the population growth of fungivorous nematodes, the effects of fungivorous nematodes on fungal biomass have been unpredictable [13, $16,21,22]$.

To enhance the proliferation and establishment of T. harzianum, hyphal biomass and fungal spores have been encapsulated in alginate pellets with an additional nutrient source such as wheat bran $[9,23]$. Hyphae rapidly grow out from the formulated alginate pellets and form networks in soil [9, 24, 25]. Bae and Knudsen [14] reported that hyphal growth of $T$. harzianum was rapid for at least 14 days in a partially sterilized soil but was rapid for only 5 days (after which growth stopped) in a nonsterile soil. They also showed that the presence of a fungivorous nematode can reduce the growth of the biocontrol fungus and adversely affect its biocontrol activity. In addition, Orr and Knudsen [26] reported that the hyphal density of $T$. harzianum peaked after 3-6 days in a nonsterile soil. Thus, there may be a specific trophic interaction between nematodes and an introduced filamentous fungus in soil, and this preypredator relationship may be a key determinant in the survival and activity of a biocontrol fungus.

In this study, we hypothesized that soil nematodes selectively feed on active hyphae of introduced filamentous fungal agents, and this selective feeding adversely affects the hyphal growth of the introduced fungi and reduces their relative fitness compared with that of indigenous fungi. In general, it is difficult to distinguish between introduced fungi and indigenous fungi. In order to monitor and assess the population change and growth of T. harzianum in natural environments, the genetic transformant $T$. harzianum ThzID1-M3, with three exogenous genes encoding green fluorescent protein (GFP), hygromycin B resistance, and $\beta$-glucuronidase, was produced [14]. The expression of these genes allow propagules of the introduced ThzID1-M3 to be successfully distinguished from indigenous fungi in a complex microbial community such as natural soil and, therefore, provides a useful tool for evaluating the hyphal growth and population change of the fungus. For instance, hyphal proliferation of an introduced agent that expresses green fluorescence can be accurately quantified in a natural soil by using epifluorescence microscopy and image analysis [26].

\section{Materials and Methods}

\section{Formulation of Trichoderma harzianum}

T. harzianum ThzID1-M3, a transformant strain of T. harzianum ThzID1, contains three exogenous genes encoding GFP, hygromycin $\mathrm{B}$ resistance, and $\beta$-glucuronidase. ThzID1 as a mycoparasitic fungus was originally isolated from field soil near Moscow, Idaho, USA. ThzID1-M3 was allowed to grow for 1 week on potato dextrose agar (PDA) plates at $25^{\circ} \mathrm{C}$, and three $1-\mathrm{cm}^{2}$ pieces from the sporulating culture were transferred to a 1-L flask containing $500 \mathrm{ml}$ of potato dextrose broth with streptomycin $(25 \mathrm{~g} / \mathrm{ml})$. Flasks were plugged with sterile cotton, placed on a rotary shaker $(120 \mathrm{rpm})$, and incubated at room temperature for 1 week with $12 \mathrm{~h}$ of light per day; subsequently, the cultures of ThzID1-M3 were strained through cheesecloth and rinsed thoroughly with sterile water. Thirty-seven grams of hyphal biomass was blended briefly with $2 \mathrm{~g}$ of wheat bran and $100 \mathrm{ml}$ of $1 \%$ aqueous sodium alginate solution. Drops of the mixture were added to $0.25 \mathrm{M}$ aqueous $\mathrm{CaCl}_{2}$, forming a pellet precipitate. The pellets were removed by straining, placed on waxed paper to air-dry at room temperature, and stored at $4^{\circ} \mathrm{C}$ before use. The pellets used were approximately $0.021 \pm 0.002 \mathrm{~g}$.

\section{Preparation of Aphelenchoides sp.}

Aphelenchoides sp., a known fungivorous nematode, was maintained on T. harzianum ThzID1 culture plates. Rhizoctonia sp. was allowed to grow on PDA for 5 days. Three $1-\mathrm{cm}^{2}$ pieces of the ThzID1 plates with Aphelenchoides sp. were transferred onto Rhizoctonia sp. culture plates and these plates were incubated for 10 days. To eliminate possible contamination by T. harzianum, three $1-\mathrm{cm}^{2}$ pieces of the Rhizoctonia sp. plates with Aphelenchoides sp. were transferred onto new Rhizoctonia sp. culture plates. After 15 days, the Aphelenchoides sp. nematodes were extracted for 2 days by using Baermann funnels. Approximately $6 \mathrm{ml}$ of extract from each funnel was placed in a test tube. For washing nematodes, $25 \mathrm{ml}$ of sterile distilled water was added to the test tube. Each tube was vigorously mixed once by hand and kept at $4^{\circ} \mathrm{C}$ overnight. The added amount of water was aspirated carefully by suctioning. The nematodes were washed once again with sterile distilled water.

\section{Soil Preparation}

Palouse silt loam soil was obtained from the University of Idaho Plant Science Farm near Moscow. The collected soil was air-dried and sieved through a 2-mm mesh prior to use. Soil analysis (performed by the University of Idaho Analytical Service Laboratory) results showed that the soil contained 20\% sand, $20 \%$ 
clay, and $60 \%$ silt by weight, and $82.2 \mu \mathrm{g}$ of plant-available iron per gram. The soil pH in soil-water (2:1) was approximately 5.9.

\section{Experimental Treatments}

Soil was adjusted to a soil moisture content of $-50 \mathrm{kPa}$ with sterile distilled water or nematode suspensions of Aphelenchoides sp. to provide nematode population levels of 0 or 10 Aphelenchoides sp. per gram of soil. The soil was mixed well to ensure that the added nematodes were uniformly distributed. The soil (300 g) was added to containers, approximately $8 \times 6.3 \times 6.3 \mathrm{~cm}$ in size. Alginate pellets of T. harzianum ThzID1-M3 were attached to plastic toothpicks by using cyanoacrylate glue. The treated toothpicks were randomly assigned to containers and placed vertically in the soil at a depth of approximately $5 \mathrm{~cm}$ (one toothpick per container). The containers were covered with lids and sealed with parafilm to maintain a relatively constant moisture level. All experiments were conducted at $22^{\circ} \mathrm{C} \pm 2{ }^{\circ} \mathrm{C}$ in a laboratory environment. There were four treatments: single pellet with 10 Aphelenchoides sp. per gram of soil; single pellet without nematodes; no pellet and 10 Aphelenchoides sp. per gram of soil; and no pellet and no nematodes. There were five replicates of each treatment at every sample time. After $0,5,10,20$, and 40 days, approximately $10 \mathrm{~g}$ of soil sampled from around a pellet was collected by using a steel corer with a diameter of $1.2 \mathrm{~cm}$. The whole experiment was repeated once.

\section{Microbial Population and Numbers of Nematodes}

Each collected soil sample was homogenized in $90 \mathrm{ml}$ of $50 \mathrm{mM}$ sodium phosphate buffer ( $\mathrm{pH}$ 7.0) on a rotary shaker (200 rpm) for $1 \mathrm{~h}$. A subsample $(1 \mathrm{ml})$ of each soil suspension was serially diluted in $9 \mathrm{ml}$ of the phosphate buffer. For enumerating total recoverable propagules of Trichoderma spp., aliquots $(0.1 \mathrm{ml})$ were spread onto agar plates of Trichoderma semiselective medium (TSM) [27]. For enumerating propagules of ThzID1-M3, aliquots $(0.1 \mathrm{ml})$ were spread onto TSM agar plates with hygromycin B $(50 \mu \mathrm{g} / \mathrm{ml})$ [14]. For total bacteria, aliquots $(0.1 \mathrm{ml})$ were spread onto tryptic soil agar plates with cycloheximide $(50 \mu \mathrm{g} / \mathrm{ml})$. The plates were incubated for 5 to 7 days at $23^{\circ} \mathrm{C}$ in an incubator and numbers of colony forming units were counted. Because filamentous fungi other than Trichoderma could be recovered from TSM agar plates, colonies belonging to Trichoderma were identified by growth on the selective medium, colony color, and morphology of conidia and conidiophores.

To enumerate the nematodes, each soil suspension was placed on four layers of tissue paper in a Baermann funnel filled with distilled water. Extracts were collected every 2 days over a 6-day period; approximately $6 \mathrm{ml}$ was obtained from each funnel and placed in a test tube. For each sample, a 1-ml aliquot was placed in a small Syracuse dish, and the total nematodes were counted by using a dissecting microscope at $32 \times$ magnification.

\section{Biomasses of Total Fungi and T. harzianum ThzID1-M3}

Fungi, total fungi, and T. harzianum ThzID1-M3 expressing GFP were quantified using epifluorescence microscopy and image analysis [26]. In brief, homogenized soil suspensions (1/100 dilution) were obtained from the soil dilution series as mentioned above. Subsamples $(1 \mathrm{ml})$ of the soil suspensions (1/100 dilution) were filtered through non-fluorescent membrane filters (Millipore MF Black filter, $0.8 \mu \mathrm{m}, 47 \mathrm{~mm}$ diameter) to quantify the biomass of ThzID1-M3. In order to microscopically observe active fungal hyphae, fungi should be stained with live cell dyes such as fluorescein diacetate (FDA). Subsamples $(1 \mathrm{ml})$ of the soil suspensions (1/100 dilution) were stained for $3 \mathrm{~min}$ at a final concentration of $10 \mu \mathrm{g} / \mathrm{ml} \mathrm{FDA} \mathrm{(Sigma-Aldrich,} \mathrm{USA)} \mathrm{and} \mathrm{filtered}$ through non-fluorescent membrane filters (Millipore MF Black filter).

Autofluorescence within the range of GFP and fluorescent FDA derivatives from soil substances such as minerals, organic debris, plant tissue, and other microbes on the membrane filters was alleviated with a triple filter cube with DAPI/FITC/TRITC (excitation wavelengths of 380-400, 470-490, and 545-565 nm; dichroic mirror wavelengths of 425-470,500-540, and $570 \mathrm{~nm}$; and barrier wavelengths of 450-465, 510-540, and 580-620 nm). Each filter was examined in its entirety (grid by grid) for any fungal segments expressing green fluorescence at 100x magnification with a Nikon Eclipse E1000 epifluorescence microscope (Nikon, USA). Images were captured by using a Hamamatsu C4742-95 digital camera and MetaMorph computer software. Every picture that included a fungal hyphal fragment was saved and analyzed to estimate the fungal biomass.

The conversion factor from a pixel of a captured image to a micrometer value at the magnification used $(100 \times)$ was calculated as $1 \mu \mathrm{m} /$ pixel, using a standard hemocytometer. On each captured image, radii and lengths of visible hyphae were quantified by using ImagePro Plus imaging software (Media Cybernetics, USA). The ThzID1-M3 and total fungal biomass estimates were calculated by applying the following formula: fungal biomass $(B)=\pi \times r^{2} \times L \times d$, where $r$ is the radius of fungal hyphae, $L$ is the length of fungal hyphae, and $d$ is the density of fungal hyphae $[28,29]$. The density of fungal hyphae was set at $0.33 \mathrm{~g} / \mathrm{cm}^{3}$.

\section{Biomass of ThzID1-M3 and Total Fungi in Heat-Treated Soil}

After soil was air-dried, it was heat-treated for $30 \mathrm{~min}$ at $80^{\circ} \mathrm{C}$ to eliminate indigenous nematodes and then allowed to stand in a room for 10 days. The soil was adjusted to a soil moisture content of $-50 \mathrm{kPa}$ with sterile distilled water and a $300-\mathrm{g}$ sample was added to each container. Alginate pellets of T. harzianum ThzID1M3 on toothpicks were placed vertically in the soil at a depth of approximately $5 \mathrm{~cm}$. The containers were covered with lids and sealed with parafilm to maintain a relatively constant moisture level. At $0,5,10,20$, and 40 days after sealing, the biomass of total fungi and ThzID1-M3 was measured.

\section{Statistical Analysis}

This study was conducted by using a completely randomized block design with five replicates. This experimental design had the following structures: (i) alginate pellet of T. harzianum ThzID1- 
M3 (two levels, 0 and 1); (ii) Aphelenchoides sp. (two levels, 0 and 10 per gram of soil); and (iii) sample times as blocks (five levels, 0 , $5,10,20$, and 40 days). Microbial populations were logarithmically transformed, and microbial biomasses and nematode populations were log-transformed $(x+1)$ prior to analysis. All analyses were performed by using SAS package 8.2 (SAS Institute Inc., USA). Analyses of variance (ANOVAs) using PROC GLM were performed for each population and biomass result, and multiple comparisons were accomplished by applying Tukey's method; significant differences were detected at the $p<0.05$ level. The Pearson correlation test was used to investigate the relationship between the nematode and bacterial populations.

\section{Results}

Effects of T. harzianum ThzID1-M3 and Aphelenchoides sp. on Nematode Populations

Addition of ThzID1-M3 significantly stimulated the population growth of nematodes $(p<0.05)$, regardless of whether Aphelenchoides sp. (10 per gram of soil) was added (Fig. 1). When ThzID1-M3 was not added, indigenous nematodes were initially at an undetectable level, and their number did not increase with time; moreover, the added Aphelenchoides sp. nematodes were undetectable at day 10, and there was no resurgence of the total nematode population (Fig. 1). ANOVA results showed that the main effects of the added ThzID1-M3 and Aphelenchoides sp. and their interactive effect were significant $(p<0.05)$. At all sample times, the nematode population densities were significantly greater in soil with the combined addition of ThzID1-M3 and Aphelenchoides sp. than with the added

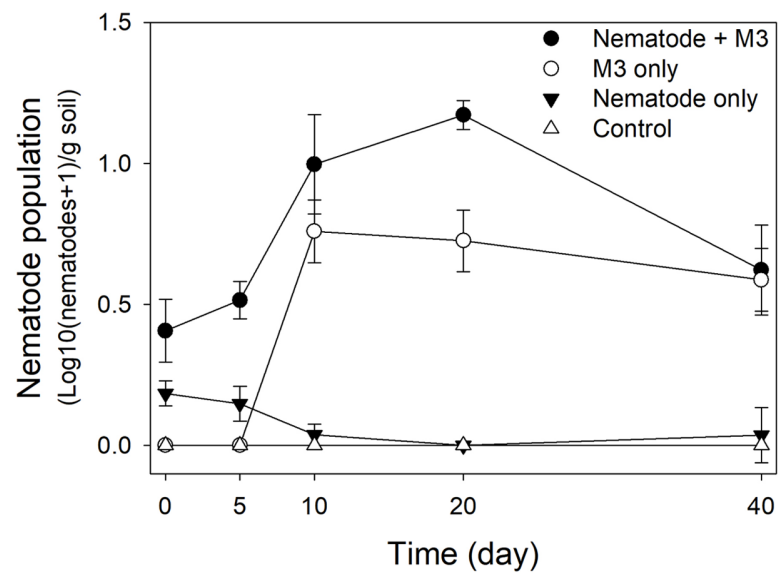

Fig. 1. Effects of Trichoderma harzianum ThzID1-M3 and Aphelenchoides sp. on nematode population in nonsterile soil. ThzID1-M3 (single alginate pellet) and/or Aphelenchoides sp. (10 adults per gram of soil) were added to the soil. Vertical bars represent \pm 1 standard error of the mean (SEM). fungus only $(p<0.05)$. There was a significant effect of sample time on nematode population $(p<0.05)$. When ThzID1-M3 was added, the numbers of recoverable nematodes increased sharply over the 5-10-day posttreatment period but slightly decreased at day 40 .

\section{Effects of ThzID1-M3 and Aphelenchoides sp. on Total Fungal Biomass}

The total fungal biomass was measured at each sample day (Fig. 2A). ANOVA results showed that the addition of ThzID1-M3 and Aphelenchoides sp. had a significant effect $(p<0.05)$ but their interactive effect was not significant $(p>0.05)$ on the total fungal biomass. There was a significant effect of sample time on the total fungal biomass $(p<0.05)$, with the total fungal biomass peaking at day 5 and then decreasing over time in all treatments. The fungal biomass was significantly greater in soil with the combined addition of ThzID1-M3 and Aphelenchoides sp. than with the added fungus only $(p<0.05)$, but was not greater than with the addition of Aphelenchoides sp. only ( $p>0.05)$. Moreover,

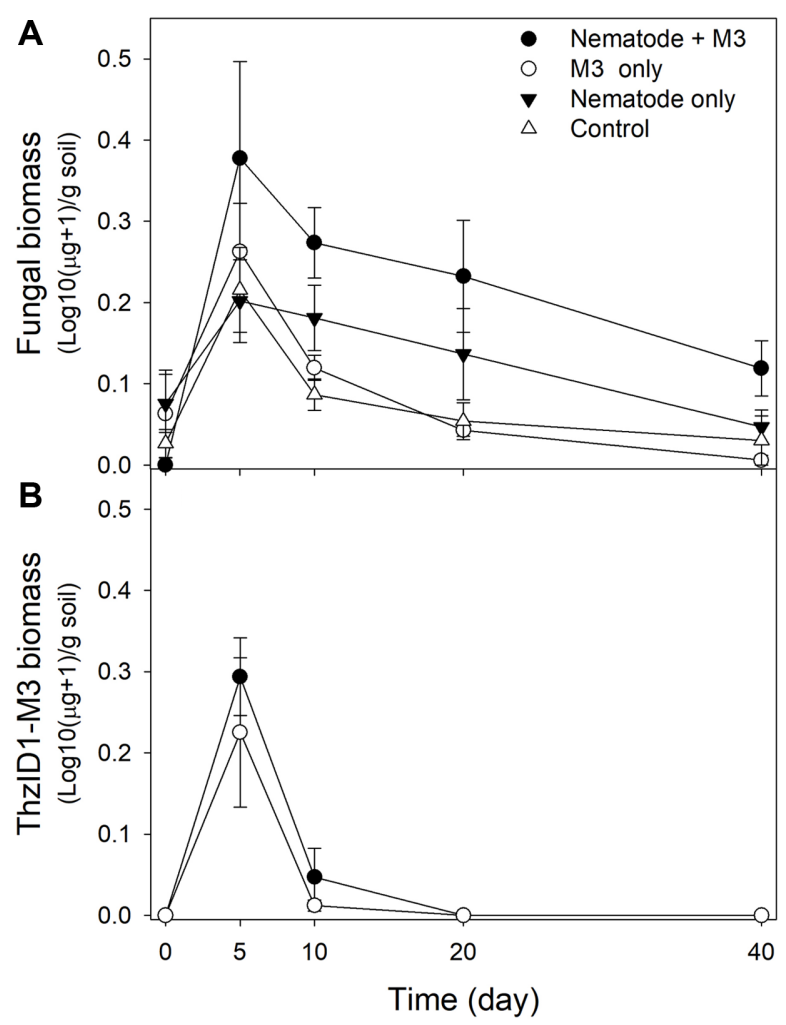

Fig. 2. Effects of T. harzianum ThzID1-M3 and Aphelenchoides sp. on the total fungal biomass (A) and ThzID1-M3 biomass (B) in nonsterile soil.

ThzID1-M3 (single alginate pellet) and/or Aphelenchoides sp. (10 adults per gram of soil) were added to the soil. Vertical bars represent \pm 1 SEM. 
the fungal biomass was not significantly greater in soil with ThzID1-M3 than in the control soil $(p>0.05)$.

\section{Effect of Aphelenchoides sp. on the Biomass and Population of ThzID1-M3}

At all sample times, there were no significant effects of the added Aphelenchoides sp. on the biomass and population of ThzID1-M3 $(p>0.05)$, but the effect of sample time was significant ( $p<0.05$ ) (Figs. $2 B$ and 3C). The ThzID1-M3 biomass peaked at day 5 , dropped sharply at day 10 , and was undetectable at day 20 (Fig. 2B).

\section{Total Fungal Biomass and ThzID1-M3 Biomass in Heat-} Treated Soil

By using heat-treated soil, we attempted to eliminate the possibility of direct effects of indigenous nematodes. In the

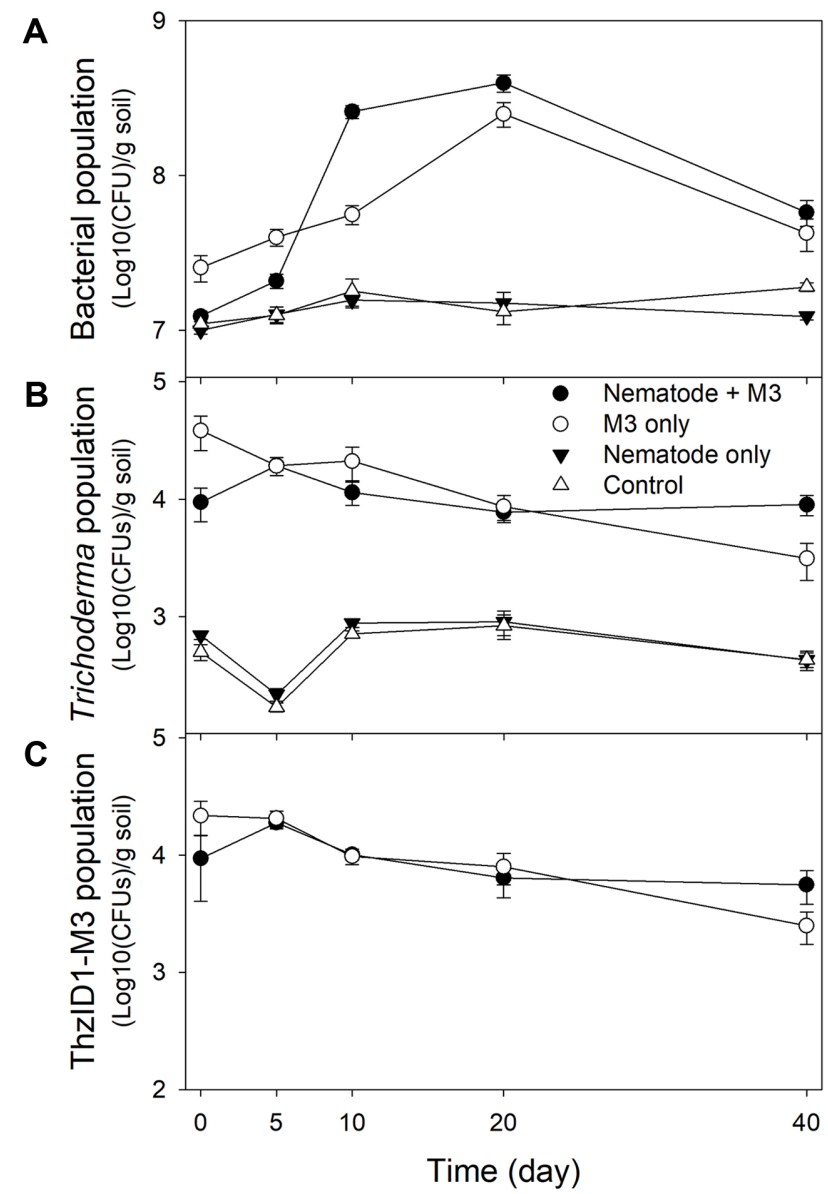

Fig. 3. Effects of T. harzianum ThzID1-M3 and Aphelenchoides sp. on microbial populations in nonsterile soil.

(A) Total bacteria; (B) total Trichoderma spp.; and (C) ThzID1-M3. ThzID1-M3 (single alginate pellet) and/or Aphelenchoides sp. (10 adults per gram of soil) were added to the soil. Vertical bars represent \pm 1 SEM. heat-treated soil, the ThzID1-M3 biomass peaked at day 10, decreased at day 20, and did not change further; whereas the total fungal biomass peaked at day 5 and maintained that level over time (Fig. 4). There was a substantial amount of ThzID1-M3 biomass over the 20-40-day post-treatment period compared with the amount in the nonsterile soil (Figs. 2B and 3).

\section{Effects of ThzID1-M3 and Aphelenchoides sp. on Microbial Populations}

Populations of Trichoderma spp., total bacteria, and ThzID1-M3 were monitored over time (Fig. 3). The added ThzID1-M3 significantly increased the Trichoderma and bacterial populations $(p<0.05)$. ANOVA results showed that the main effects of the added ThzID1-M3 and Aphelenchoides sp. and the effect of their interaction were significant on bacterial populations $(p<0.05)$. There was a significant effect of sample time on bacterial populations $(p<0.05)$. The bacterial population increased significantly at day 20 and then decreased over time in soil with ThzID1M3 alone, but it did not increase in soil without the added ThzID1-M3. The bacterial population density was significantly greater in the treatment with the added fungus and nematodes than with the added fungus alone $(p<0.05)$. There was a significant correlation between the nematode and bacterial population densities $(p<0.05)$.

The addition of ThzID1-M3 and Aphelenchoides sp. had a significant effect on the recoverable Trichoderma population $(p<0.05)$, but there was no significant effect of their interaction on the Trichoderma population $(p>0.05)$. The

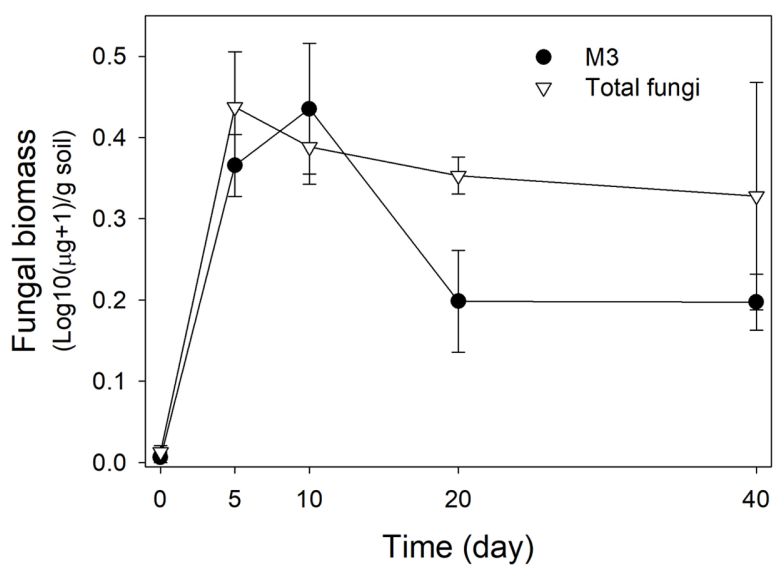

Fig. 4. Trichoderma harzianum ThzID1-M3 biomass (0) and total fungal biomass $(\nabla)$ in sterile soil.

Soil was heat-treated $\left(80^{\circ} \mathrm{C}, 30 \mathrm{~min}\right)$ to eliminate nematodes, after which a single alginate pellet of T. harzianum ThzID1-M3 was added. Vertical bars represent \pm 1 SEM. 
population densities of Trichoderma were significantly greater in ThzID1-M3 treatments than in treatments without the fungus $(p<0.05)$. The increased population densities of Trichoderma resulted from ThzID1-M3 propagules from pellets. There was a significant effect of sample time $(p<0.05)$. The numbers of ThzID1-M3 recoverable propagules decreased gradually over time.

\section{Discussion}

Soil nematodes selectively fed on the actively growing hyphae of the introduced biocontrol fungus T. harzianum ThzID1-M3 compared with the level of feeding on hyphae of indigenous fungi. This trophic interaction strongly reduced the hyphal biomass and relative fitness of the introduced fungal biocontrol agent. Therefore, the establishment of introduced fungal biocontrol agents in natural environments may depend on trophic interactions between nematodes and the hyphae of the fungal agents.

The only remarkable population growth of nematodes took place in the presence of the introduced fungus ThzID1-M3. It is probable that ThzID1-M3 hyphae from alginate pellets are an easily obtainable food for fungivorous nematodes such as Aphelenchoides sp. [14]. The ThzID1-M3 biomass increased rapidly until day 5 , followed by a rapid reduction, whereas the total fungal biomass in all treatments peaked on day 5 and then decreased over time. The added ThzID1-M3 significantly increased the recoverable number of nematodes but did not affect the total fungal biomass. In contrast, the addition of Aphelenchoides sp. (10 per gram of soil) significantly increased the fungal biomass $(p<0.05)$, but the increased fungal biomass had no effect on the population growth of nematodes; moreover, the added nematodes rapidly disappeared when ThzID1-M3 was not added. Hyphae of total fungi were likely to be homogeneously distributed in the soil, whereas hyphae of ThzID1-M3 were highly localized around the alginate pellets. Radial growth of ThzID1-M3 from pellets was reported to be rapid for approximately 5-7 days and then almost stops at a maximum radius in nonsterile soil [14, 25]. In addition, the hyphal density of T. harzianum ThzID1 declined exponentially with distance from the pellets [9].

The mass of ThzID1-M3 hyphae originating from the alginate pellets stimulated the population growth of nematodes near the pellets. Bae and Knudsen [14] observed that there were many indigenous nematodes and their eggs associated with ThzID1-M3 hyphae adjacent to pellets in a soil. We observed that fungivores made up the majority of the nematode population that was increased by a fungal pellet in another experiment (data not shown). Fungivorous nematodes presumably were the main contributors to the total nematode population in this experiment. The number of recoverable nematodes increased rapidly for 5-10 days after the hyphal biomass of ThzID1-M3 peaked. This rapid increase in nematode population size likely resulted from a sudden hatching of nematode eggs, stimulated by the presence of ThzID1-M3 hyphae. For instance, the common genera of fungivores in agricultural soil are Aphelenchoides, Aphelenchus, Tylenchus, and Ditylenchus, and it takes from 5 to 20 days or more to complete their life cycle [15-18, 30]. Hyphae of ThzID1-M3 were undetectable by day 20 in nonsterile soil, while there were significant levels of ThzIDM3 hyphal density over the course of the experiment in heat-treated soil with inactivated indigenous nematodes. The active population of fungivorous nematodes was responsible for reducing the hyphal density of the introduced fungus in the nonsterile soil.

There are conflicting reports on the effect of fungivorous nematodes on the fungal biomass [13, 21, 22]. For example, Mikola and Setälä [22] found that populations of Aphelenchoides sp. were stimulated by fungi, but the nematode populations did not regulate total fungal biomass in a microcosm comprising a sterilized mixture of leaf litter and humus with 10 bacterial species, 10 fungal species, and Aphelenchoides sp. Nieminen and Setälä [21] observed a similar result, in that the number of Aphelenchoides sp. was increased by Cladosporium herbarum, and the nematode population increased the biomass of the fungus after a certain time in their microcosm. In contrast, Chen and Ferris [16] reported that the soilborne fungus Rhizoctonia solani increased the number of Aphelenchoides composticola, whereas the nematode reduced the biomass of the fungus in a column with sand and organic substrates. Bae and Knudsen [14] observed that when alginate pellets of T. harzianum ThzID1-M3 and Aphelenchoides sp. (10 per gram of soil) were added to a heat-treated soil, the added fungus significantly increased the number of Aphelenchoides sp., which in turn significantly reduced the radial growth of the fungus from the pellets. In addition, Ingham et al. [13] observed a similar result from the rhizosphere microcosm, but not from the non-rhizosphere and nonplant microcosms. In this study, we found that hyphae of total fungi that were homogeneously distributed did not stimulate nematode population growth, whereas hyphae of ThzID1-M3, which were densely localized adjacent to the pellets, significantly increased the nematode population level. Thus, the increase in grazing intensity of nematodes around alginate pellets reduced the hyphal density of 
ThzID1-M3. The results indicate that the interaction of the fungivorous nematode and fungal biomass can be predictable at sites where fungal hyphae are densely localized. It should be noted that fungivorous nematodes exerted selective feeding on the active fungal hyphae of localized ThzID1-M3, without a particular preference for the fungus over other filamentous fungi.

Our preliminary results showed that the added Aphelenchoides sp. nematodes (10 per gram of soil) reduced the radial growth of $T$. harzianum ThzID1-M3 from the pellets [14], but we observed no effect on the hyphal density of ThzID1-M3 in this study. Mean measurements of ThzID1-M3 were greater in soil with the added nematodes than in soil without them. Fungivorous nematodes physically penetrate the hyphal walls of fungi with their stylet in order to use the cell cytoplasm as a nutrient. The physical damage to ThzID1-M3 hyphae by the added nematodes may stimulate the hyphae to undergo hyperbranching in the initial phase of growth from the pellets. When fungal hyphae are physically damaged, the loss of cytoplasm from the damaged hyphae is reduced by the rapid occlusion of septal pores, after which the hyphae rapidly form hyphal branches just below the septa segregating the damaged sections [31, 32]. Ujcová et al. [33] observed that hyphae of Aspergillus niger formed densely branched mycelia under high physical stress conditions, such as that associated with high turbine speed in a fermenter. Nematode population increases stimulated by ThzID1-M3 were responsible for the increased levels of bacterial populations. Nematodes feeding on fungal hyphae may be able to stimulate an increase in the numbers of soil bacteria, which can rapidly decompose dead fungal hyphae [34]. Furthermore, bacterial cells may be transported either internally or externally by nematodes to a substrate-rich microhabitat, and excretory products of nematodes may provide substrates or inorganic nutrients for bacterial growth [13]. The increased population density of bacteria may adversely affect the activity and growth of fungi in soil microhabitats [35] through, at least in part, competition for nutrient sources and antagonistic actions.

\section{Acknowledgments}

This work was supported by a 2-Year Research Grant of Pusan National University.

\section{Conflict of Interest}

The authors have no financial conflicts of interest to declare.

\section{References}

1. Knudsen GR, Eschen DJ, Dandurand LM, Bin L. 1991. Potential for biocontrol of Sclerotinia sclerotiorum through colonization of sclerotia by Trichoderma harzianum. Plant Dis. 75: 446-470.

2. Elad Y. 2000. Trichoderma harzianum T39 preparation for biocontrol of plant diseases - control of Botrytis cinerea, Sclerotinia sclerotiorum and Cladosporium fulvum. Biocontrol Sci. Technol. 10: 499-507.

3. Hadar Y, Harman GE, Taylor AG. 1984. Evaluation of Trichoderma koningii and T. harzianum from New York soils for biological control of seed rot caused by Phythium spp. Phytopathology 74: 106-110.

4. Elad Y, Chet I, Katan J. 1980. Trichoderma harzianum: a biocontrol agent effective against Sclerotium rolfsii and Rhizoctonia solani. Phytopathology 70: 119-121.

5. Dandurand LM, Mosher RD, Knudsen GR. 2000. Combined effects of Brassica napus seed meal and Trichoderma harzianum on two soilborne plant pathogens. Can. J. Microbiol. 46: 10511057.

6. Chet I, Baker R. 1980. Induction of suppressiveness to Rhizoctonia solani in soil. Phytopathology 70: 994-998.

7. Wells HD, Bell DK, Jaworski CA. 1972. Efficacy of Trichoderma harzianum as a biocontrol for Sclerotium rolfsii. Phytopathology 62: $442-447$.

8. Sivan A, Chet I. 1989. The possible role of competition between Trichoderma harzianum and Fusarium oxysporum on rhizosphere colonization. Phytopathology 79: 198-203.

9. Knudsen GR, Bin L. 1990. Effects of temperature, soil moisture, and wheat bran on growth of Trichoderma harzianum from alginate pellets. Phytopathology 80: 724-727.

10. Sohlenius B. 1980. Abundance, biomass and contribution to energy flow by soil nematodes in terrestrial ecosystems. Oikos 34: 186-194.

11. Procter DLC. 1990. Global overview of the functional roles of soil-living nematodes in terrestrial communities and ecosystems. J. Nematol. 22: 1-7.

12. Bongers T, Ferris H. 1999. Nematode community structure as a bioindicator in environmental monitoring. Trends Ecol. Evol. 14: 224-228.

13. Ingham RE, Trofymow JA, Ingham ER, Coleman DC. 1985. Interactions of bacteria, fungi, and their nematode grazers: effects on nutrient cycling and plant growth. Ecol. Monogr. 55: 119-140.

14. Bae Y-S, Knudsen GR. 2001. Influence of a fungus-feeding nematode on growth and biocontrol efficacy of Trichoderma harzianum. Phytopathology 91: 301-306.

15. Freckman DW, Caswell EP. 1985. The ecology of nematodes in agroecosystem. Annu. Rev. Phytopathol. 23: 275-296.

16. Chen J, Ferris H. 1999. The effects of nematode grazing on nitrogen mineralization during fungal decomposition of organic matter. Soil Biol. Biochem. 31: 1265-1279. 
17. Neher DA, Weicht TR, Savin M, Görres JH, Amador JA. 1999. Grazing in a porous environment. 2. Nematode community structure. Plant Soil 212: 85-99.

18. Yeates GW, Wardle DA, Watson RN. 1999. Responses of soil nematode populations, community structure, diversity and temporal variability to agricultural intensification over a seven-year period. Soil Biol. Biochem. 31: 1721-1733.

19. Yeates GW, Wardle DA, Watson RN. 1993. Relationships between nematodes, soil microbial biomass and weedmanagement strategies in maize and asparagus cropping systems. Soil Biol. Biochem. 25: 869-876.

20. Steinberger Y, Sarig S. 1993. Response by soil nematode populations and the soil microbial biomass to a rain episode in the hot, dry Negev desert. Biol. Fertil. Soils 16: 188-192.

21. Nieminen JK, Setälä H. 2001. Bacteria and microbial-feeders modify the performance of a decomposer fungus. Soil Biol. Biochem. 33: 1703-1712.

22. Mikola J, Setälä H. 1998. No evidence of trophic cascades in an experimental microbial-based soil food web. Ecology 79: 153-164.

23. Fravel DR, Marois JJ, Lumsden RD, Connick WJ. 1985. Encapsulation of potential biocontrol agents in an alginateclay matrix. Phytopathology 75: 774-777.

24. Knudsen GR, Eschen DJ, Dandurand LM, Wang ZG. 1991. Method to enhance growth and sporulation of pelletized biocontrol fungi. Appl. Environ. Microbiol. 57: 2864-2867.

25. Bae Y-S, Knudsen GR. 2000. Cotransformation of Trichoderma harzianum with $\beta$-glucuronidase and green fluorescent protein genes provides a useful tool for monitoring fungal growth and activity in natural soil. Appl. Environ. Microbiol. 66: 810-815.

26. Orr KA, Knudsen GR. 2004. Use of green fluorescent protein and image analysis to quantify proliferation of Trichoderma harzianum in nonsterile soil. Phytopathology 94: 1383-1389.

27. Papavizas GC. 1981. Survival of Trichoderma harzianum in soil and in pea and bean rhizospheres. Phytopathology 71: 121-125.

28. van Veen JA, Paul EA. 1979. Conversion of biovolume measurements of soil organisms, grown under various moisture tensions, to biomass and their nutrient content. Appl. Environ. Microbiol. 37: 686-692.

29. Daniel O, Schönholzer F, Zeyer J. 1995. Quantification of fungal hyphae in leaves of deciduous trees by automated image analysis. Appl. Environ. Microbiol. 61: 3910-3918.

30. Yeates GW, Bongers T, Goede RG, Freckman DW, Georgieva SS. 1993. Feeding habits in soil nematode families and genera - an outline for soil ecologists. J. Nematol. 25: 315-331.

31. Trinci APJ, Collinge AJ. 1974. Occlusion of the septal pores of damaged hyphae of Neurospora crassa by hexagonal crystals. Protoplasma 80: 57-67.

32. Watters MK, Griffiths AJF. 2001. Tests of a cellular model for constant branch distribution in the filamentous fungus Neurospora crassa. Appl. Environ. Microbiol. 67: 1788-1792.

33. Ujcová E, Fencil Z, Musílková M, Seichert L. 1980. Dependence of release of nucleotides from fungi on fermentor turbine speed. Biotechnol. Bioeng. 22: 237-241.

34. Lockwood JL, Filonow AB. 1981. Responses of fungi to nutrient-limiting conditions and to inhibitory substances in natural habitats, pp. 1-61. In Alexander M (ed.). Advances in Microbial Ecology, 1st Ed. Plenum Press, New York and London.

35. Eastburn DM, Butler EE. 1988. Microhabitat characterization of Trichoderma harzianum in natural soil: evaluation of factors affecting distribution. Soil Biol. Biochem. 20: 547-553. 